\title{
Lnc-M2 controls M2 macrophage differentiation via the PKA/CREB pathway
}

\author{
Yuxiang Chen ${ }^{1}$, Hanzhao $\mathrm{Li}^{2}$, Tengteng Ding ${ }^{2}$, Jinbang $\mathrm{Li}^{3}$, Yuanbin Zhang ${ }^{2}$, Jianguo \\ Wang $^{2}$, Xu Yang ${ }^{2}$, Tuotuo Chong ${ }^{2}$, Yufei Long ${ }^{2}$, Xiaoming Lyu ${ }^{4}$, Fei Gao ${ }^{5}$, and xin Li $^{2}$ \\ ${ }^{1}$ Southern Medical University Nanfang Hospital \\ ${ }^{2}$ Shenzhen Hospital of Southern Medical University \\ ${ }^{3}$ The Sixth Affiliated Hospital of Guangzhou Medical University \\ ${ }^{4}$ The Third Affiliated Hospital, Southern Medical University \\ ${ }^{5}$ Mayo Clinic Division of Gastroenterology and Hepatology
}

April 28, 2020

\begin{abstract}
LncRNAs play an indispensable role in the process of M1 macrophage via regulating the development of macrophages and their responses to bacterial pathogens and viral infections. However, there are few studies on the lncRNA-mediated functions and regulatory mechanisms of M2 macrophage polarization. In this study, we found a number of differentially expressed lncRNAs between human monocyte derived M0 and M2 macrophages according to array analysis and qRT-PCR validation. The lncRNA RP11-389C8.2 (we named lnc-M2 in this study) was observed to be highly expressed in M2 macrophages. In Situ Localization and Quantification Analysis showed that lnc-M2 was expressed in the nucleus and cytosolic compartments of M2 macrophages. Notably, lnc-M2 knockdown enhanced the phagocytic ability of M2 macrophages. Ulteriorly, the results of RNA-Protein interaction experiments indicated that protein kinase A (PKA) was a lnc-M2 associated RNA-binding protein (RBP). Western blot showed that p-CREB, a well-known key downstream transcription factor of PKA, was lowly phosphorylated in lnc-M2silencing M2 macrophages. Furthermore, we found that transcriptional factor STAT3 promoted lnc-M2 transcription along with the up-regulation of epigenetic histone modification markers at the lnc-M2 promoter locus, indicating that STAT3 activated lnc-M2 and eventually facilitated the process of M2 macrophage differentiation via the PKA/CREB pathway. Collectively, our date provide evidence that the transcription factor STAT3 can promote the transcription of lnc-M2 and facilitated the process of M2 macrophage differentiation via the PKA/CREB pathway. This study highlights a novel mechanism underlying the M2 macrophage differentiation.
\end{abstract}

\section{Hosted file}

manuscript-2020-03-31. doc available at https://authorea.com/users/307725/articles/438716-lncm2-controls-m2-macrophage-differentiation-via-the-pka-creb-pathway 
(a)

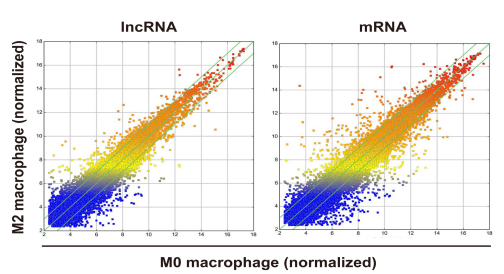

(c)

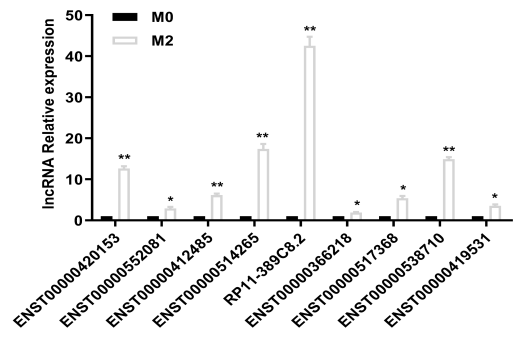

(d)

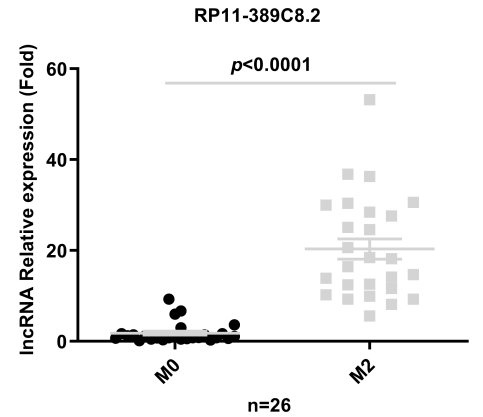

(b)

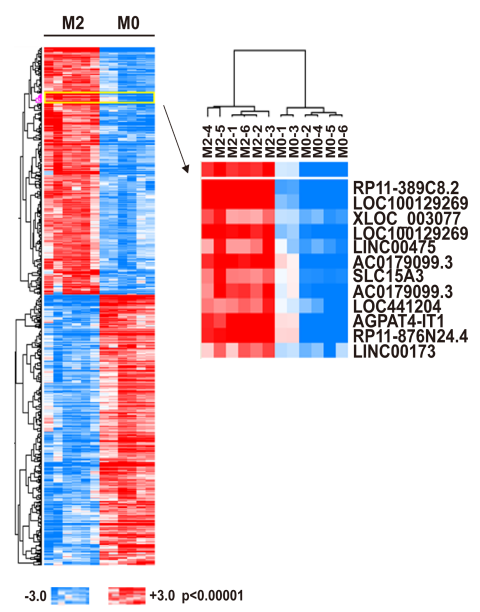

(e)

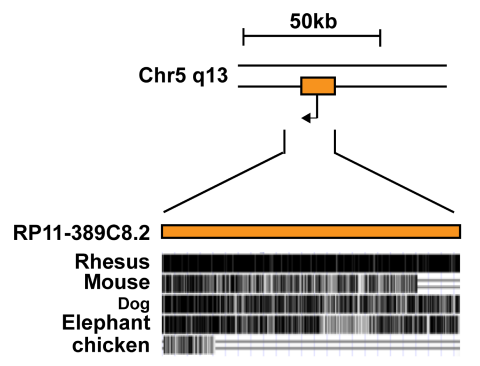


(a)
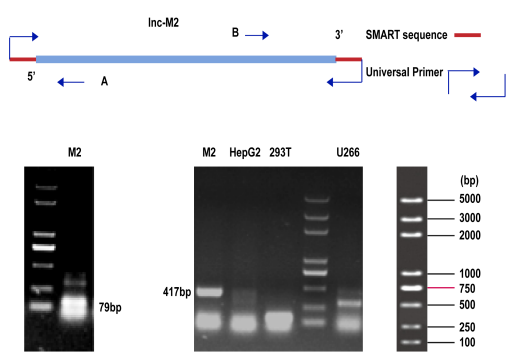

(c)

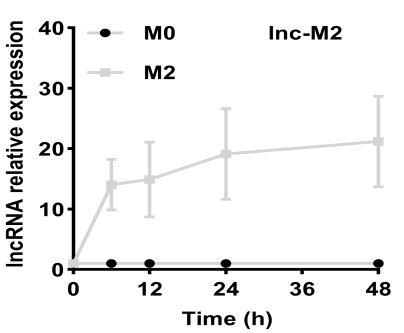

(e)

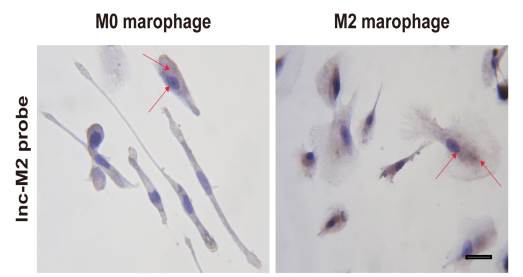

(b)

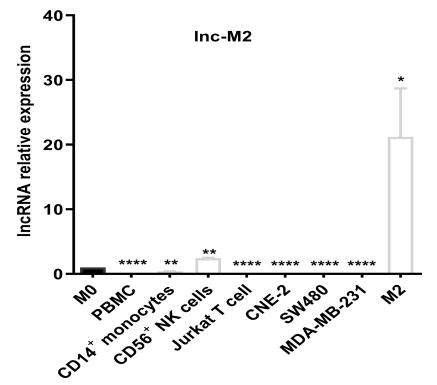

(d)

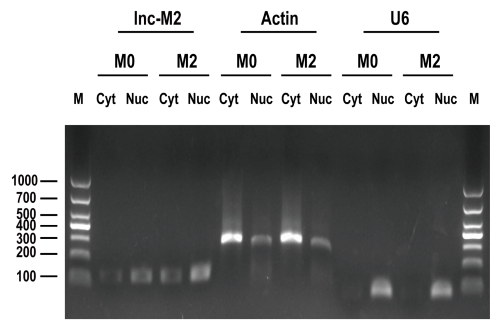

(f)

NCBI ORF Finder (Open Reading Frame Finder)

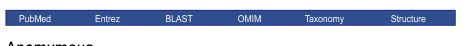

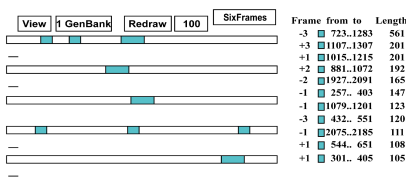


(a)

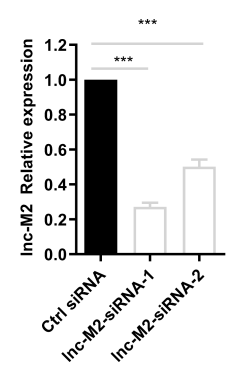

(c)

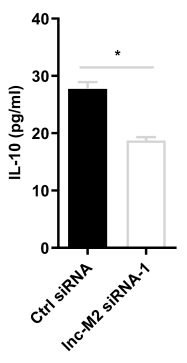

(f)

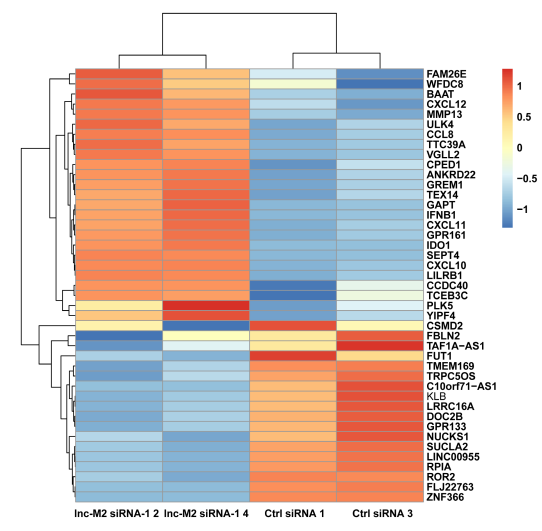

(b)

(d)

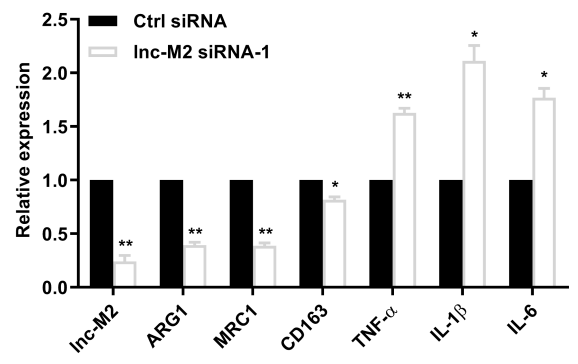

(e)
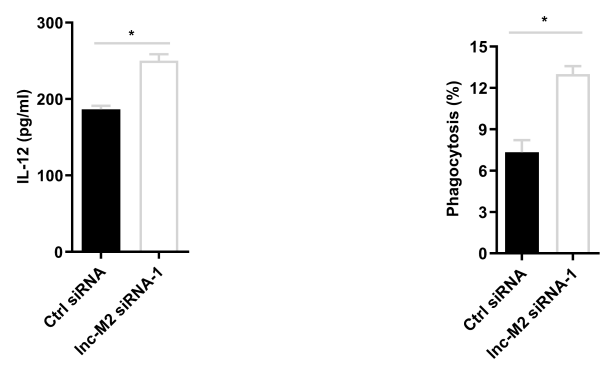

(g)

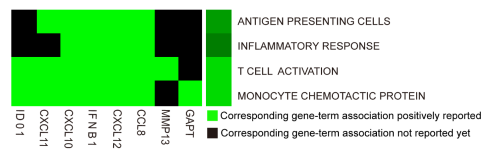

(h)

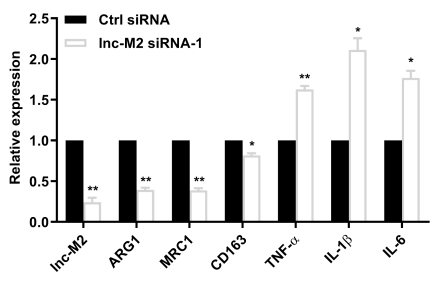


(a)

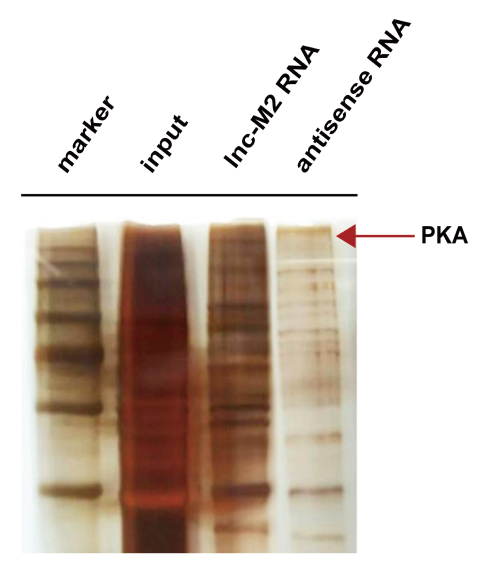

(d)

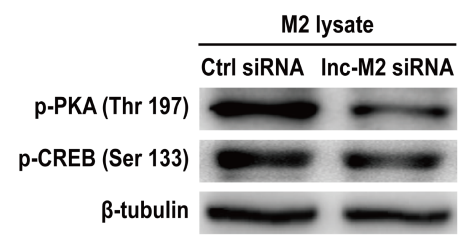

(b)

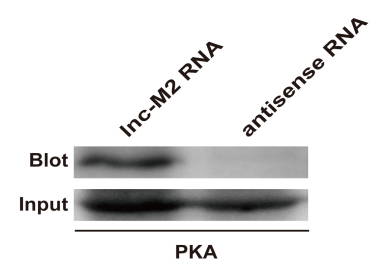

(c)

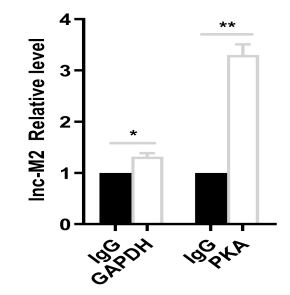

(e)

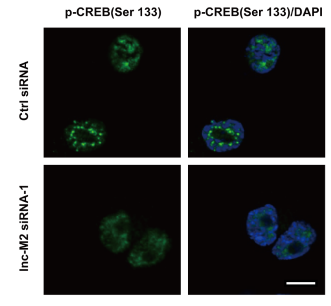

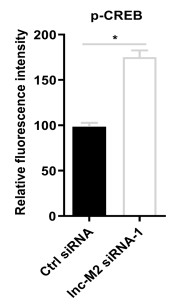



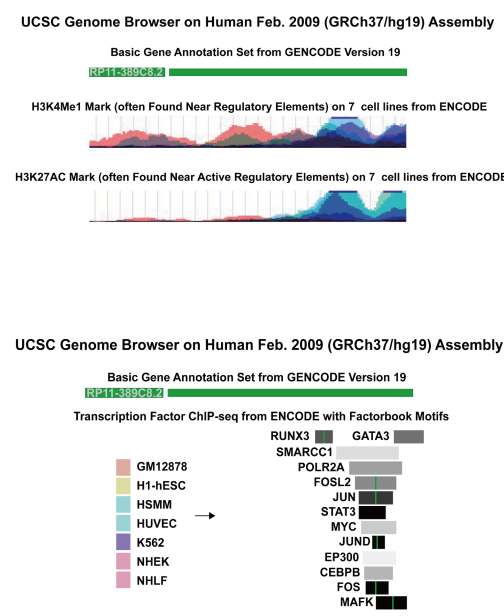

(c)

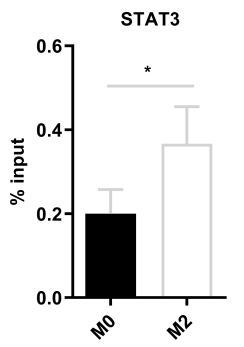

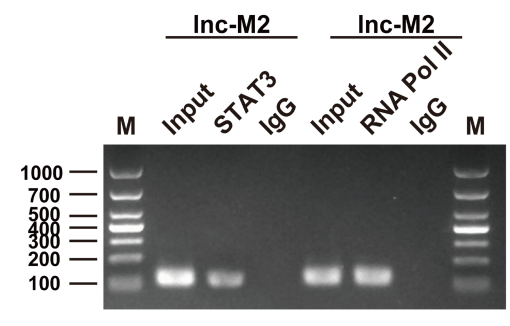

(e)

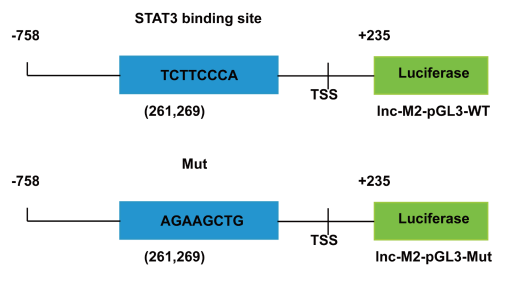

(f)

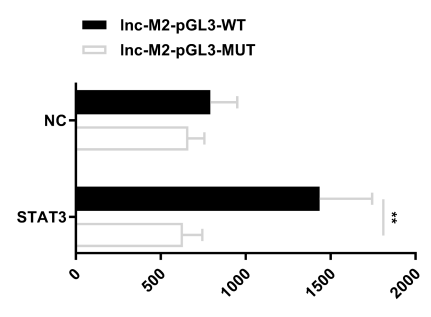

Inc-M2 promoter activity 